\title{
Controlling Protein-Protein Interactions through Metal Coordination: Assembly of a 16-Helix Bundle Protein
}

\author{
Eric N. Salgado, Jasmin Faraone-Mennella, and F. Akif Tezcan \\ University of California, San Diego, Department of Chemistry and Biochemistry, 9500 Gilman Dr., \\ La Jolla, CA 92093
}

\begin{abstract}
Protein-protein interactions (PPI's) are central to nearly all processes within cells, whether they are formed transiently in dynamic networks or permanently in macromolecular assemblies. There has been considerable progress towards our understanding of how proteins recognize their partners and how the energetics of their interactions are tuned. ${ }^{1}$ Nevertheless, the ability to predict or interfere with natural PPI's or engineer new ones remains a great challenge, owing to the fact that protein-protein docking processes are guided by the superposition of many weak, non-covalent bonds that spread over large and often flexible surfaces. Our goal is to utilize the strength, directionality and selectivity of metal-ligand interactions to control PPI's, thereby achieving specificity and affinity without requiring extensive binding surfaces. We describe here the $\mathrm{Zn}$-mediated construction of a 16-helix architecture comprising four copies of cytochrome $c b_{562}\left(c y t c b_{562}\right)$, a 4-helix bundle heme protein. Our results demonstrate that the self-organization of this macromolecule is controlled by metal coordination, with little or no thermodynamic bias from specific protein-protein contacts.
\end{abstract}

There are several factors that make $c y t c b_{562}$ a good model system for investigating metalmediated PPI's. It has a highly stable helical-bundle fold that is further strengthened through the engineering of covalent heme-polypeptide linkages into the parent protein, cyt $b_{562}{ }^{2,3}$ As a result, its structure is not perturbed by modifications on its surface. The all- $a$-helical makeup of $c y t c b_{562}$ leads to uniform surface features that facilitate the introduction of metal binding motifs. In addition, $c y t c b_{562}$ has a rigid, roughly cylindrical shape, ideal for use as a building block for protein superstructures.

An earlier examination of the crystal structure of cyt $c b_{562}$ revealed that the protein molecules are associated in pairs within the crystal lattice, despite being monomeric even at millimolar concentrations. ${ }^{3}$ The pairing is mediated by a crystal packing contact formed by the antiparallel alignment of Helix 3's (residues 56-80) from individual molecules. Based on this non-functional packing arrangement, we envisioned that metal-mediated interactions between cyt $c b_{562}$ monomers could be forged through the incorporation of metal coordinating motifs onto the Helix 3 surface. To this end, we engineered a variant ( $\mathrm{His}^{4}$ $c b_{562}$ ) featuring two di-histidine motifs near the $\mathrm{N}$ - and $\mathrm{C}$-termini of this helix at positions

tezcan@ucsd.edu.

Supporting Information Available: Materials and methods for protein expression/purification/characterization, experimental details for DLS, NMR and SV measurements, crystallographic data collection and refinement statistics. 
59/63 and 73/77, which are located directly opposite each other in the crystal lattice and could potentially participate in interprotein metal coordination. Such di-His motifs placed at $i$ and $i+4$ positions on an a-helix can coordinate metal ions with high affinity, and have been widely used for the assembly of metalloproteins and peptides. ${ }^{4-7}$ In addition, two Asp residues at positions 60 and 74 contained within each di-His motif were left intact in order to increase the likelihood of stable metal coordination. ${ }^{8}$

Our studies on metal-mediated PPI's have focused on late firstrow transition metal ions. $\mathrm{Zn}$ (II), in particular, should have a high binding affinity for the di-His motifs on $\mathrm{His}^{4}-c b_{562}$ and exhibit rapid ligand exchange, which should prevent the formation of kinetically trapped protein-protein complexes. While dynamic light scattering measurements suggested the formation of multimeric $\mathrm{His}^{4}-\mathrm{cb}_{562}$ structures upon $\mathrm{Zn}$ (II) addition, aggregates were formed at metal:protein ratios exceeding $2: 1$, even at protein concentrations below $100 \mu \mathrm{M}$. These protein aggregates were readily dissolved upon EDTA addition or lowering the solution $\mathrm{pH}$ below 6, indicating that $\mathrm{Zn}$-His coordination is responsible for protein oligomerization. At high $\mathrm{Zn}$ loadings, both di-His clamps are likely charged with metal, which can lead to multiple modes of interprotein coordination, and thereby, aggregation. To capture the structures of any homogenous protein assemblies, we screened for crystals of $\mathrm{His}^{4}-\mathrm{cb}_{562}$ at low Zn loadings, and obtained diffraction quality ones at less than 2:1 metal:protein ratios.

The 2.9- $\AA$ data reveal a unique quaternary structure (PDB ID: 2QLA) stabilized by four $\mathrm{Zn}$ (II) ions, wherein four $\mathrm{His}^{4}-\mathrm{cb}_{562}$ monomers form two interlaced V-shaped dimers to yield a 16-helix bundle (Figure 1). ${ }^{9}$ Each V-shape is formed by the parallel alignment of two cyt $c b_{562}$ molecules related by a non-crystallographic two-fold symmetry axis, forming a $\sim 37^{\circ}$ interprotein angle. The two V's, on the other hand, are wedged into one another in an antiparallel fashion. Both the formation of the V's and their interlacing are achieved entirely by interprotein $\mathrm{Zn}$-coordination. Each $\mathrm{Zn}$ shares an identical distorted tetrahedral coordination environment with ligands from three monomers (Figure 1c): the 73/77 di-His clamp from one molecule holds the $\mathrm{Zn}$ in a bidentate fashion, coordination of Asp74 from a second molecule stabilizes the V-arrangement between the two, and coordination of His63 from a third molecule locks the two V's together. This striking arrangement suggests that the formation of the protein assembly and metal coordination should be highly cooperative. Interestingly, the 59/63 di-His clamp is not utilized for bidentate coordination despite the likelihood that it has a similar $\mathrm{Zn}$ affinity as the $73 / 77$ couple. The His59 sidechain is, in fact, H-bonded to Thr31 across the interface, but not involved in metal coordination (Figure 1b). The discrimination by $\mathrm{Zn}$ between the two di-His motifs is in accord with the dynamic self-assembly of the $c y t c b_{562}$ superstructure: the lability of $\mathrm{Zn}$ likely permits the exploration of different coordination geometries, resulting in the formation of the thermodynamically most stable quaternary structure.

The interfaces between the four $\mathrm{His}^{4}-\mathrm{c} b_{562}$ monomers feature a large number of polar interactions and bury a surface area exceeding $5000 \AA^{2}$. While the surface of $c y t c b_{562}$ is not optimized for self-association and the interfacial contacts are likely to be non-specific, they may collectively impart sufficient thermodynamic driving force to bias the formation of the observed assembly. To probe the existence of any metal-independent pre-organization between the $\mathrm{His}^{4}-\mathrm{cb}_{562}$ monomers and to examine their metal-dependent oligomerization 
behavior in solution, we carried out NMR and sedimentation velocity (SV) experiments. Indicative of a monomeric species, the 1D-proton-NMR spectra of $\mathrm{His}^{4}-\mathrm{cb} \mathrm{b}_{562}$ at high protein concentrations $(>1 \mathrm{mM})$ feature significant chemical shift dispersion. Upon addition of one equivalent of $\mathrm{Zn}$, the peaks broaden considerably, as expected from the formation of a highorder oligomer. In order to quantitatively determine the hydrodynamic properties of this oligomer, we utilized pulsed field gradient (PFG) diffusion NMR spectroscopy. ${ }^{10}$ These experiments yielded diffusion coefficients of $1.17 \times 10^{-6}$ and $0.785 \times 10^{-6} \mathrm{~cm}^{2} / \mathrm{s}$ for the protein in the absence and presence of one equivalent of $\mathrm{Zn}$, respectively, with corresponding Stokes radii of $17.6 \AA$ and $26 \AA$. The $\sim 9-\AA$ expansion is consistent with the formation of a tetramer.

The molecular mass distributions of $\mathrm{His}^{4}-\mathrm{cb}_{562}$ species determined by SV measurements under different solution conditions are shown in Figure 2. In the absence of $\mathrm{Zn}$, the protein is monomeric at all concentrations tested (up to $400 \mu \mathrm{M}$ ), with a single maximum at $\sim 11500$ $\mathrm{Da}$ (12328 Da actual). Upon addition of $\mathrm{Zn}$, two new peaks centered at $\sim 22 \mathrm{kDa}$ and $\sim 50$ $\mathrm{kDa}$ emerge at the expense of the monomeric species. The $22-\mathrm{kDa}$ species presumably corresponds to either one of the two possible dimeric halves of the 16-helix bundle: a) the Vshaped His ${ }^{4}-c b_{562}$ dimer (e.g., Mol1 and Mol3 in Figure 1) held together by two $\mathrm{Zn}$ ions with His73/77 and Asp74 coordination, or b) the antiparallel dimer (e.g., Mol1 and Mol4) with His $73 / 77$ and His 63 coordination. These two species can further dimerize into the observed 16-helix bundle structure through His63-Zn or Asp74-Zn coordination, respectively, and become the predominant species at high protein concentrations.

Metal coordination chemistry has been used successfully for directing the formation of discrete non-biological supramolecular complexes. ${ }^{11}$ We have demonstrated here that protein building blocks with non-interacting surfaces can be assembled into self-healing superstructures through metal coordination. Such chemical control of protein-protein interactions paves the way for the generation of new biomaterials and manipulation of cellular processes.

\section{Supplementary Material}

Refer to Web version on PubMed Central for supplementary material.

\section{Acknowledgement}

We thank Drs. Arnold L. Rheingold and Antonio DiPasquale for their help with X-ray data collection, and the Komives Lab, Dr. Andrew Herr (University of Cincinnati) and Dr. Xuemei Huang for their assistance with SV and NMR experiments. This work was supported by the University of California, San Diego, a Hellman Faculty Scholar Award (F.A.T.), N.I.H. Molecular Biophysics Training Grant (E.N.S), and N.S.F. (Instrumentation Grant 0634989 to A.L.R.).

\section{References}

1. a Shoemaker BA, Panchenko AR. PLos Comput. Biol. 2007; 3:595-601.b Vizcarra CL, Mayo SL. Curr. Opin. Chem. Biol. 2005; 9:622-626. [PubMed: 16257567] c Kortemme T, Baker D. Curr. Opin. Chem. Biol. 2004; 8:91-97. [PubMed: 15036162] d DeLano WL. Curr. Opin. Struct. Biol. 2002; 12:14-20. [PubMed: 11839484] e Sheinerman FB, Norel R, Honig B. Curr. Opin. Struct. Biol. 2000; 10:153-159. [PubMed: 10753808] 
2. Allen JWA, Barker PD, Ferguson SJ. J. Biol. Chem. 2003; 278:52075-52083. [PubMed: 14534316]

3. Faraone-Mennella J, Tezcan FA, Gray HB, Winkler JR. Biochemistry. 2006; 45:10504-10511. [PubMed: 16939202]

4. Arnold FH, Haymore BL. Science. 1991; 252:1796-1797. [PubMed: 1648261]

5. Ghadiri MR, Choi C. J. Am. Chem. Soc. 1990; 112:1630-1632.

6. Handel TM, Williams SA, Degrado WF. Science. 1993; 261:879-885. [PubMed: 8346440]

7. Krantz BA, Sosnick TR. Nat. Struct. Biol. 2001; 8:1042-1047. [PubMed: 11694889]

8 .

The only other His residue (102) in this variant is coordinated to the heme iron in the protein core and thus unable to participate in PPI's.

9.

Though only at 2.9 - $\AA$ resolution, the crystallographic data quality is sufficient to determine the architecture of the protein assembly, the configurations of most sidechains, and the metal coordination.

10. Altieri AS, Hinton DP, Byrd RA. J. Am. Chem. Soc. 1995; 117:7566-7567.

11. a Lehn JM. Science. 2002; 295:2400-2403. [PubMed: 11923524] b Leininger S, Olenyuk B, Stang PJ. Chem. Rev. 2000; 100:853-907. [PubMed: 11749254] c Caulder DL, Raymond KN. Acc. Chem. Res. 1999; 32:975-982.d Holliday BJ, Mirkin CA. Angew. Chem., Int. Ed. Eng. 2001; 40:2022-2043.e Ockwig NW, Delgado-Friedrichs O, O'Keeffe M, Yaghi OM. Acc. Chem. Res. 2005; 38:176-182. [PubMed: 15766236] 
a)

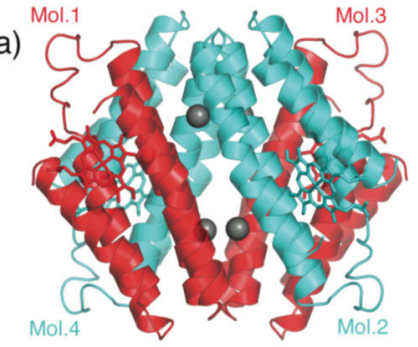

b)

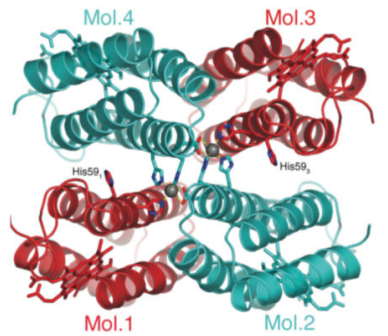

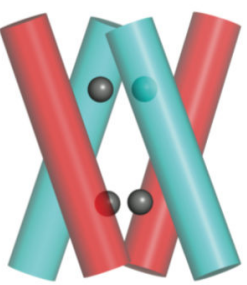

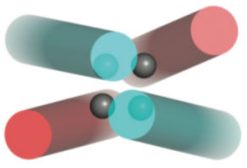

c)

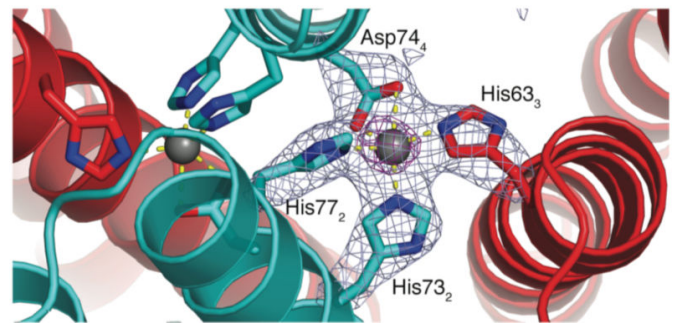

Figure 1.

Crystal structure of the $4 \mathrm{Zn}-4 \mathrm{His}^{4}-c b_{562}$ assembly. Pairs of protein molecules that form the V-shaped dimers are colored alike. Zn ions are shown as grey spheres. (a) View of the assembly parallel to the non-crystallographic twofold axis and the corresponding cylindrical representation of Helix 3's involved in Zn coordination; (b) view down the noncrystallographic twofold axis; (c) close-up view of the Zn coordination environment and simulated-annealing $F_{o}-F_{c}$ omit electron density map (grey, $4 \sigma$; purple, $12 \sigma$ ). The parent molecules for metal ligands are shown in subscripts. 


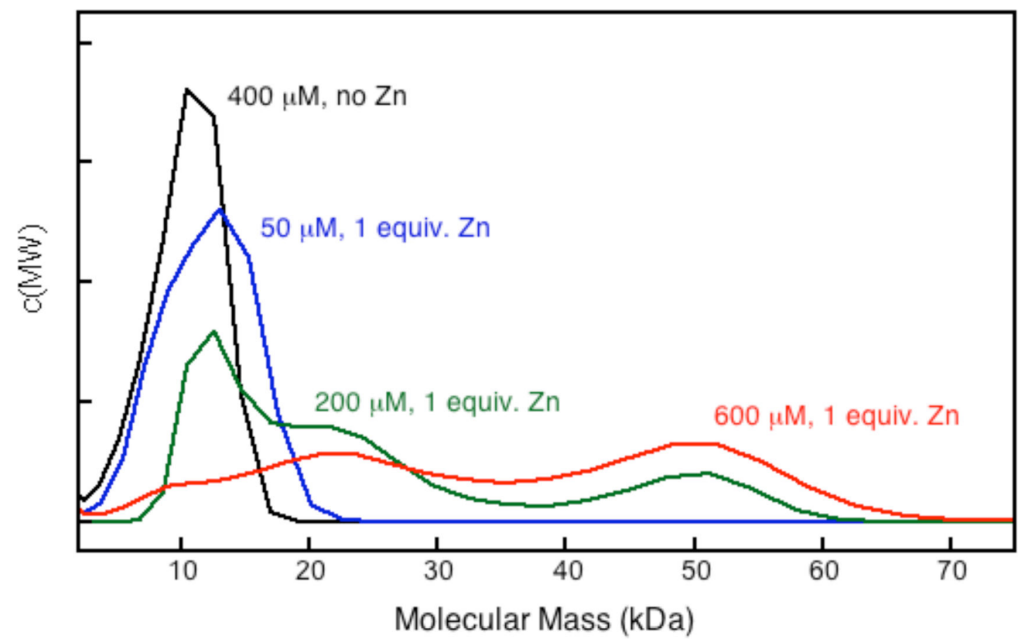

Figure 2.

Molecular mass distributions of $\mathrm{His}^{4}-\mathrm{cb}_{562}$ species determined by sedimentation velocity experiments. The distributions are normalized with respect to the area covered under the curves. 